\title{
SARS-CoV-2 Serologic Assays in Control and Unknown Populations Demonstrate the Necessity of Virus Neutralization Testing
}

\author{
Jennifer A. Rathe, ${ }^{1,2, a}$ Emily A. Hemann, ${ }^{1, a, b}$ Julie Eggenberger, ${ }^{1}$ Zhaoqi Li, ${ }^{3}$ Megan L. Knoll, ${ }^{1}$ Caleb Stokes, ${ }^{1,2}$ Tien-Ying Hsiang, ${ }^{1}$ Jason Netland, ${ }^{1}$ \\ Kennidy K. Takehara, ${ }^{1}$ Marion Pepper, ${ }^{1}$ and Michael Gale $\mathrm{Jr}^{1}$
}

'Department of Immunology, Center for Innate Immunity and Immune Disease, University of Washington, Seattle, Washington, USA, ${ }^{2}$ Department of Pediatric Infectious Disease, Seattle Children's Hospital/University of Washington, Seattle, Washington, USA, and ${ }^{3}$ Department of Statistics, University of Washington, Seattle, Washington, USA

Background. To determine how serologic antibody testing outcome links with virus neutralization of severe acute respiratory syndrome coronavirus 2 (SARS-CoV-2), we evaluated individuals for SARS-CoV-2 antibody level and viral neutralization.

Methods. We compared serum Ig levels across platforms of viral antigens and antibodies with 15 positive and 30 negative SARSCoV-2 controls followed by viral neutralization assessment. We then applied these platforms to a clinically relevant cohort of 114 individuals with unknown histories of SARS-CoV-2 infection.

Results. In controls, the best-performing virus-specific antibody detection platforms were SARS-CoV-2 receptor binding domain (RBD) IgG (sensitivity $87 \%$, specificity $100 \%$, positive predictive value [PPV] 100\%, negative predictive value [NPV] $94 \%$ ), spike IgG3 (sensitivity 93\%, specificity 97\%, PPV 93\%, NPV 97\%), and nucleocapsid protein (NP) IgG (sensitivity 93\%, specificity 97\%, PPV 93\%, NPV 97\%). Neutralization of positive and negative control sera showed 100\% agreement. Twenty individuals with unknown history had detectable SARS-CoV-2 antibodies with 16 demonstrating virus neutralization. Spike IgG3 provided the highest accuracy for predicting serologically positive individuals with virus neutralization activity (misidentified $1 / 20$ unknowns compared to 2/20 for RBD and NP IgG).

Conclusions. The coupling of virus neutralization analysis to a spike IgG3 antibody test is optimal to categorize patients for correlates of SARS-CoV-2 immune protection status.

Keywords. SARS-CoV-2; serologic; immunity; two-tiered testing; neutralization; antibody.

Severe acute respiratory syndrome coronavirus 2 (SARSCoV-2) has infected millions of people globally. Several serological assays to detect SARS-CoV-2 antibodies have been developed but their utility is hindered by limited sensitivity and specificity, and unclear correlation with viral neutralization [1-3]. Current interpretation of antibody test results is difficult given that individuals with virus-specific antibodies may not exhibit neutralization activity. False positives from any single assay also confound clinical interpretation serologic test results, warranting coupled analyses of virus-specific antibody titer and virus neutralization activity.

For other well-studied viral respiratory infections and associated vaccine efficacy, established threshold antibody titers

Received 30 September 2020; editorial decision 22 December 2020; accepted 23 December 2020; published online December 24, 2020.

aj. A. R. and E. A. H. contributed equally.

${ }^{b}$ Current affiliation: Department of Microbial Infection and Immunity, Ohio State University, Columbus, Ohio, USA.

Correspondence: Michael Gale, Jr, PhD, Department of Immunology and Director, Center for Innate Immunity and Immune Disease, Building E (SLU 3.1), 750 Republican Street, Box 35809, Seattle, WA 98109 (mgale@uw.edu).

The Journal of Infectious Diseases ${ }^{\circledR} \quad$ 2021;223:1120-31

(C) The Author(s) 2020. Published by Oxford University Press for the Infectious Diseases Society of America. All rights reserved. For permissions, e-mail: journals.permissions@oup.com. DOI: 10.1093/infdis/jiaa797 correlate with protective immunity from symptomatic infection and this relationship is likely to hold true for SARS-CoV-2 [413]. The majority of SARS-CoV-2-infected individuals produce neutralizing antibodies $[1,14,15]$. There have been rare cases of reinfection described despite $>9$ months of SARS-CoV-2 outbreaks and spread across the world. The reinfection cases lacked assessment of SARS-CoV-2-specific immunity prior to reinfection in that antibody titers and SARS-CoV-2-specific lymphocyte responses were not measured [16-18]. While the presence of neutralizing antibodies against SARS-CoV-2 may serve as a correlate of protective immunity, the longevity and threshold titer level of protective antibodies is unknown.

The main problem facing assessment of immune correlates of protection against SARS-CoV-2 is consensus on an accurate, high-throughput testing strategy. Comparisons across antibody testing platforms reveal that no single test performs with $100 \%$ sensitivity and specificity [1, 2, 19-22]. Further, no single test has consistently predicted viral neutralization with the detection of any particular anti-SARS-CoV-2 antibody type. These less than ideal test characteristics likely reflect a time-dependent decrease in the correlative relationship between antibody levels and the strength of viral neutralization [3, 14, 23, 24]. Antibody test platforms differ in the antibody isotypes detected (immunoglobulin $\mathrm{G}[\mathrm{IgG}]$, IgM, IgA, etc.); the immune system 
production of isotypes is time dependent and is variably related to viral neutralization $[25,26]$. An accurate and consistent SARS-CoV-2 antibody-testing platform has not been identified even when using ideal, control populations [14, 15, 27, 28]. Few studies have evaluated test performance in populations where past infection status and time from infection are unknown nor have such cohorts undergone coupled evaluation of antibody titer and viral neutralization activity [29]. Evaluation of both control and unknown populations in a comprehensive manner is needed to determine an accurate, consistent testing strategy to identify individuals with correlates of viral neutralization and protective immunity to SARS-CoV-2.

In our study we compare combinations of 4 viral antigens and 5 human antibody isotype enzyme-linked immunosorbent assay (ELISA) platforms in control and unknown exposure populations coupled to classic viral neutralization studies, revealing that a specific combination approach of antibody isotype and neutralization activity of virus-specific antibody best determines exposure and possible protection to SARS-CoV-2.

\section{METHODS}

\section{Sample Collection}

Venipuncture collected 6-10 mL of blood in EDTA blood collection tubes and spun at 1000 relative centrifugal force for 10 minutes. Plasma was separated, inactivated in a $56^{\circ} \mathrm{C}$ water bath for 1 hour, and stored at $-80^{\circ} \mathrm{C}$.

\section{Enzyme-Linked Immunosorbent Assay}

ELISA assays were performed as previously described [28]. Briefly, high-binding plates (ThermoScientific) were coated with SARS-CoV-2 receptor binding domain (RBD) [28], SARSCoV-2 spike [30], or UV-inactivated SARS-CoV-2 (WA-1; BEI Resources) and incubated overnight at $4^{\circ} \mathrm{C}$. Plates were blocked in phosphate-buffered saline with Tween 20 plus $3 \%$ milk for 1 hour at room temperature. Three-fold serial dilutions of plasma were added to plates in biological duplicates. Samples and the positive control spike-binding antibody CR3022 (ab273073; Abcam) were included on plates with IgG antibody binding. Following 2-hour incubation and washes, anti-human secondary antibodies conjugated to horseradish peroxidase were diluted 1:3000 and added to plates: IgG (31410; Thermofisher), IgG1 (9054; Southern Biotech), IgG3 (9210; Southern Biotech), IgM (A6907; Sigma), and IgA (A0295; Sigma). To recapitulate this study, we recommend use of the same secondary antibodies, given potential differences in performance of antibodies from different commercial sources. Following 1-hour incubation and washes, SigmaFast $o$-phenylenediamine dihydrochloride was added to plates. Ten minutes later, $2 \mathrm{M} \mathrm{H}_{2} \mathrm{SO}_{4}$ was added to wells, stopping the reaction, and plates read at an absorbance of $490 \mathrm{~nm}$ (BioTek Epoch). Optical density (OD) values for each sample dilution were plotted and area under the curve (AUC) was calculated using Prism. AUC analyses perform more accurately to inform outcomes compared to endpoint titer; we designed our experiments to apply AUC analyses [31, 32].

\section{Plaque Reduction Neutralization Test}

Plaque reduction neutralization test (PRNT) analyses were performed as previously described [33]. Briefly, 4-fold serial dilutions of heat-inactivated plasma was mixed 1:1 with 600 plaque-forming unit/mL SARS-CoV-2 WA-1 (BEI resources) in Dulbecco's phosphate buffered saline (Fisher Scientific) plus $0.3 \%$ cold water fish skin gelatin (Sigma) and incubated for 30 minutes at $37^{\circ} \mathrm{C}$. The virus plasma mixture was added in duplicate, along with virus and mock controls, to Vero cells (American Type Culture Collection) in 12-well plates and incubated for 1 hour at $37^{\circ} \mathrm{C}$. Following adsorption, plates were washed with DPBS and overlayed with a 1:1 mixture of $2.4 \%$ Avicel RC-591 (FMC) plus $2 \times$ minimum essential medium (ThermoFisher) supplemented with $4 \%$ heat-inactivated fetal bovine serum and penicillin/streptomycin (Fisher Scientific). Plates were incubated for 2 days at $37^{\circ} \mathrm{C}$. Overlay was removed and plates were washed with DPBS and fixed in 10\% formaldehyde (Sigma-Aldrich) in DPBS for 30 minutes at room temperature. Plates were washed again with DPBS and stained with $1 \%$ crystal violet (Sigma-Aldrich) in 20\% EtOH (Fisher Scientific). Plaques were enumerated and percent neutralization was calculated as 100 minus the number of plaques in serum plus virus dilution wells divided by the number of plaques in virus only control wells times 100. PRNT $_{50}$ and PRNT $_{80}$ values are shown as inverse serum dilution and were determined by calculating the $50 \%$ and $80 \%$ sigmoidal interpolation of the percent neutralization of the samples in Prism. $R^{2}$ values were determined using a nonlinear regression fit in Prism.

\section{University of Washington Nucleocapsid Protein Assay}

Plasma samples were run on the Abbott Architect instrument following the Abbott SARS-CoV-2 IgG assay instructions. Qualitative results and index values reported by the instrument were used in analysis. Values $\geq 1.4$ were considered a positive result.

\section{Statistics}

We compared age and sex distributions of the group B subjects to the population of the greater Seattle area from US Census estimates. Pearson $\chi^{2}$ test was used for sex comparisons and the Kolmogorov-Smirnov test for age comparisons. Weights were constructed for over and under sampling for age and sex by taking the ratio of the proportion of the Seattle Census versus the group B proportion. A prevalence estimate was calculated with weight adjustments (Supplementary Material). Fisher exact test was used to compare the rate of positive neutralizing antibodies against SARS-CoV-2 between exposed and unexposed groups. The values for sensitivity, specificity, positive predictive value (PPV), and negative predictive value (NPV) for the ELISA platforms and PRNT were calculated using Prism 
software. $P$ values were calculated with Fisher exact test. The $95 \%$ confidence intervals (CIs) were calculated using the hybrid Wilson/Brown method.

\section{Human Participants}

This study was conducted under University of Washington institutional review board (number 000098108). The study protocol was reviewed and approved prior to enrollment of any subjects. Subjects were provided with information about the study, risks associated, and how their privacy would be protected. To enroll in the study each subject provided verbal understanding and written consent.

\section{RESULTS}

To evaluate the presence of SARS-CoV-2-specific antibodies and neutralization capability, subjects were enrolled from the greater Seattle area for plasma collection and antibody testing. Positive controls included 15 symptomatic reverse transcription polymerase chain reaction (RT-PCR) SARS-CoV-2-positive individuals enrolled around 21 days after the positive PCR test result. All of the positive controls had mild symptoms and none were hospitalized (Supplementary Material). Thirty negative controls were obtained from a blood bank as deidentified plasma collected prior to 1 December 2019. Unknown samples consisted of 2 cohorts: group A and group B. Group A included 14 subjects with known exposure to confirmed SARS-CoV-2 infected, symptomatic individuals. Group B subjects were randomly recruited from the community with unknown exposure to or infection with SARS-CoV-2. Serum samples were collected from 26 March to 15 April 2020. Positive and negative control plasma samples were assessed by ELISAs based on 3 SARS-CoV-2 antigens: RBD, spike [30], and full SARS-CoV-2 UV-inactivated viral particles (Figure 1A). Serial dilutions of each sample generated AUC values plotted with associated subject cohorts. Antibody-positive samples were designated as any samples with an AUC above the mean +3 SDs of the AUCs
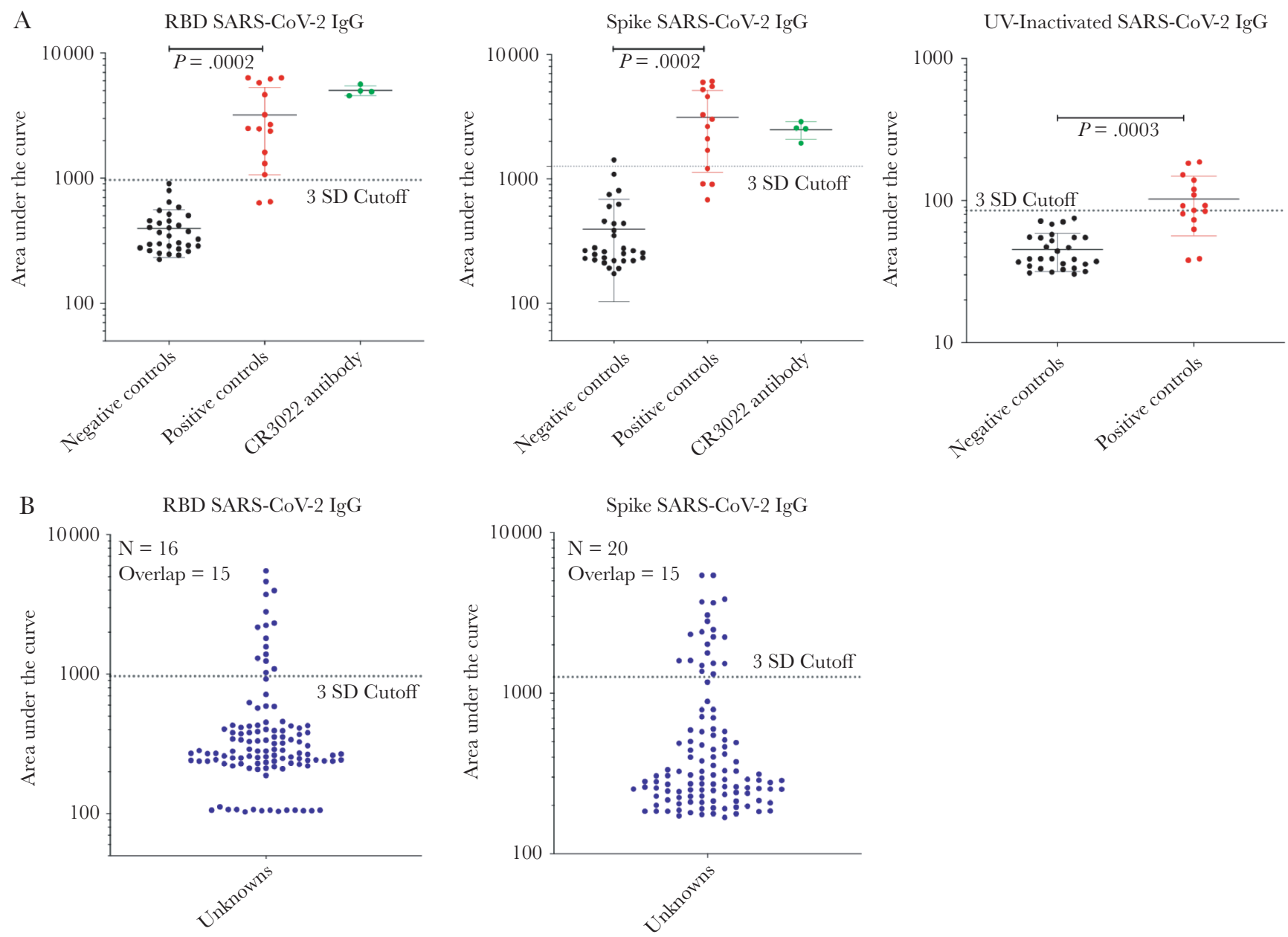

Figure 1. Comparison of ELISA IgG platforms. A, Positive and negative control serum sample total lgG antibody recognition of SARS-CoV-2 RBD, spike protein, and UV-inactivated SARS-CoV-2. B, Unknown serum samples total IgG RBD and spike assays. Mean \pm SD, Student $t$ tests for comparisons of mean of groups. Dotted lines represent cutoff of 3 SD from the mean of the negative control samples to designate "positive" antibody samples. Abbreviations: ELISA, enzyme-linked immunosorbent assay; IgG, immunoglobulin G; RBD, receptor-binding domain; SARS-CoV-2, severe acute respiratory syndrome coronavirus 2. 
of the negative control samples [28]. RBD and spike viral antigen of total IgG ELISAs demonstrated the clearest separation between positive and negative controls. Given the comparatively improved identification of control samples by RBD and spike ELISAs, no further testing of the UV-inactivated whole SARS-CoV-2 virus platform were performed. Calculations for sensitivity, specificity, PPV, NPV, and associated $95 \%$ CI were determined based on the negative and positive control samples (Table 1). RBD IgG outperformed spike IgG in all measured parameters including specificity, sensitivity, PPV, and NPV. Unknowns assessed for IgG reactivity against RBD and spike identified 16 and 20 positive samples, respectively. Fifteen of the positive samples were identified by both platforms.

We next assessed test performance of a range of antibody isotypes against SARS-CoV-2. For the positive and negative control samples, IgM reactivity was decreased compared to IgG for both RBD and spike (Figure 2A). Comparison of assay performance measures between IgG and IgM assays for RBD and spike yielded decreased sensitivity, specificity, PPV, and NPV for the IgM assays (Table 1). Analyses of the unknowns (group $A$ and $B$ ) with the IgM assays identified fewer positive samples compared to their IgG counterpart. However, all of the positives that were identified on the IgM assays were also identified on the IgG RBD and spike assays with 1 exception. All positive and negative controls as well as any unknowns identified as positive for IgG antibody titers against RBD or spike were additionally assessed for IgG1, IgG3, and IgA spike-specific antibodies (Figure 2B). IgG3 demonstrated the highest sensitivity and NPV while IgG1 and IgA had relatively superior specificity and PPV. The spike IgG3 platform detected positive reactivity in 15 of the 20 unknown samples identified as positive by the $\operatorname{IgG}$ $\mathrm{RBD}$ and spike platforms.

We tested our samples using a Food and Drug Administration (FDA) emergency use authorized ELISA based on SARS-CoV-2 nucleocapsid protein (NP) platform to identify NP-specific IgG responses. The NP IgG platform resulted in similar separation of positive and negative control subjects compared to RBD IgG, spike IgG, and spike IgG3 (Figure 2C). The NP IgG platform demonstrated similar sensitivity, specificity, PPV, and NPV compared to spike IgG3 and improved sensitivity compared to both RBD and spike IgG (Table 1). The NP IgG platform identified 14 unknowns as antibody-positive all of which were also identified by the RBD IgG, spike IgG, and spike IgG3 platforms.

PRNT analysis against SARS-CoV2/WA-1 isolate was used to assess antibody function via virus neutralization activity (Figure 3). PRNT was performed on the following samples: all positive controls, 10 negative control samples (5 with "antibodypositive" cutoff designations), any RBD/spike/NP IgG identified "positive" unknowns, and 10 unknown samples near the cutoff but technically "negative." For positive control subjects, we assessed the correlation between $\mathrm{PRNT}_{80}$ reciprocal dilutions and ELISA AUC quantitative values (Figure 3A). RBD-specific IgG ELISA results showed the strongest correlation between the magnitude of ELISA antibody signal to the strength of neutralization followed by $>$ spike IgG $>\mathrm{NP}>$ spike IgG3. We established a cutoff for detectable neutralization for unknown samples based on positive and negative control PRNT results (Supplementary Material File 1, Figures C and D). Detectable neutralization sample cut offs require (1) end point dilution levels $3 \mathrm{SD}$ greater than the mean value for negative control samples, and (2) neutralization titers present at both $\mathrm{PRNT}_{50}$ and $\mathrm{PRNT}_{80}$. Figure 3B demonstrates $\mathrm{PRNT}_{80}$ values in the left panel, with samples meeting the neutralization criteria in the right panel; 12 of the 14 group A subjects and 4 of the group B subjects showed detectable neutralization. The 10 unknown samples near the ELISA cutoffs but designated as negative were also negative for virus neutralization.

SARS-CoV-2 PRNT results provide key context to interpret ELISA results and functional evidence of viral neutralization. Table 1 illustrates the sensitivity, specificity, PPV, and NPV measurements based on the positive and negative control subjects' antibody reactivity to each of the ELISA platform assays. Sensitivity is superior for spike IgG3 and NP IgG; therefore, these 2 platforms will identify more true positive samples compared to RBD and spike IgG. Specificity is best for RBD IgG, spike IgG1, and spike IgA platforms resulting in better identification of true negatives. Overall, 3 platforms have the most desirable testing characteristics

Table 1. Summary of ELISA Platform Performance Using Positive and Negative Control Samples

\begin{tabular}{|c|c|c|c|c|}
\hline Platform & Sensitivity, $\%$ (95\% CI) & Specificity, \% (95\% CI) & PPV, \% (95\% Cl) & NPV, $\%(95 \% \mathrm{Cl})$ \\
\hline RBD IgG & 87 (62-98) & $100(89-100)$ & $100(77-100)$ & 94 (80-99) \\
\hline RBD IgM & 73 (48-89) & $97(83-100)$ & $92(65-100)$ & 88 (73-95) \\
\hline Spike IgG & $67(42-85)$ & $97(83-100)$ & $91(62-100)$ & $85(70-94)$ \\
\hline Spike IgM & $60(36-80)$ & $97(83-100)$ & $90(60-100)$ & 83 (67-92) \\
\hline Spike IgG1 & $71(45-88)$ & $100(87-100)$ & $100(72-100)$ & $88(73-95)$ \\
\hline Spike lgG3 & $93(70-100)$ & $97(83-100)$ & $93(70-100)$ & $97(83-100)$ \\
\hline Spike $\lg A$ & 67 (42-85) & 100 (89-100) & $100(72-100)$ & 86 (71-94) \\
\hline$N P \lg G$ & $93(70-100)$ & $97(83-100)$ & $93(70-100)$ & 97 (83-100) \\
\hline
\end{tabular}

Abbreviations: Cl, confidence interval; ELISA, enzyme-linked immunosorbent assay; IgA, immunoglobulin A; IgG, immunoglobulin G; IgM, immunoglobulin M; NP, nucleocapsid protein; NPV, negative predictive value; PPV, positive predictive value; RBD, receptor binding domain. 

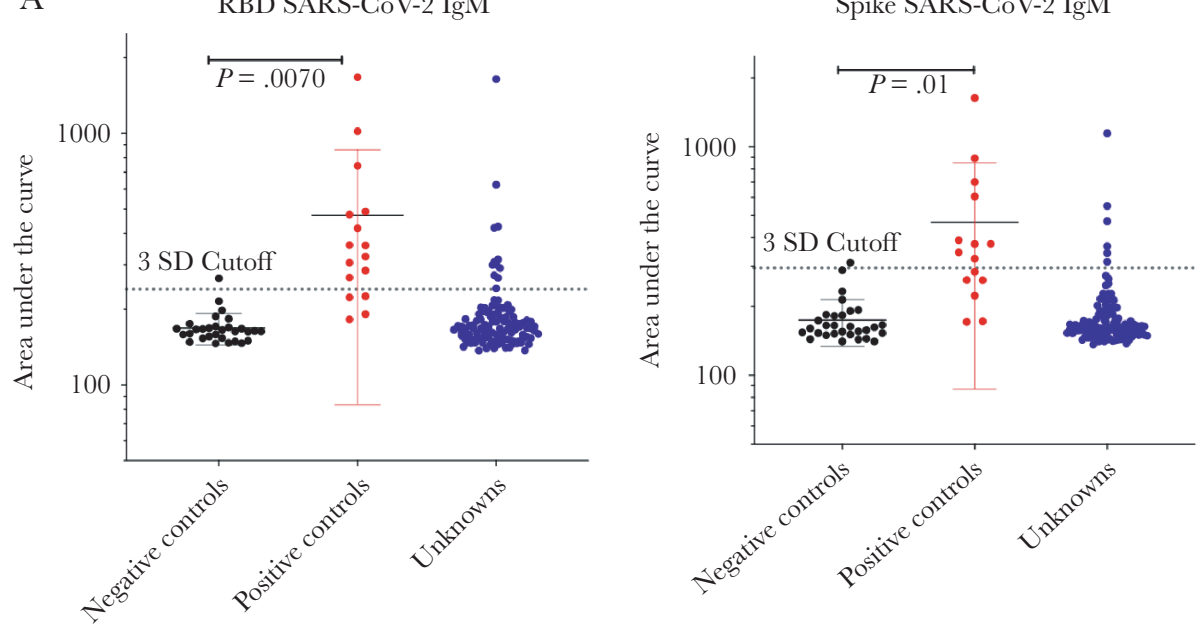

B

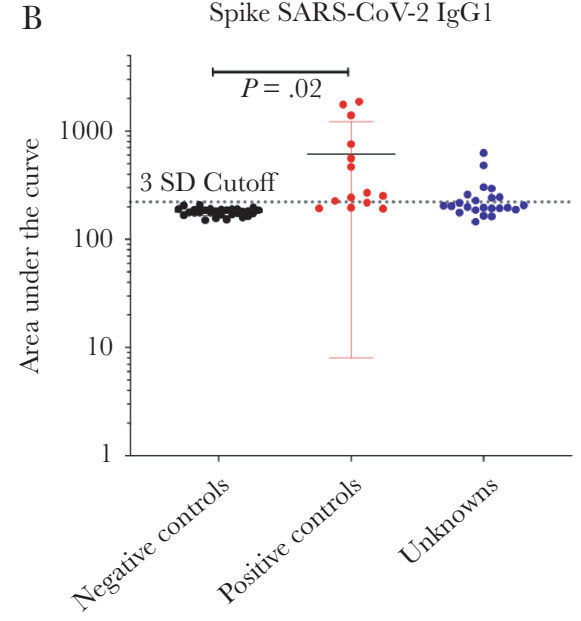

Spike SARS-CoV-2 IgA

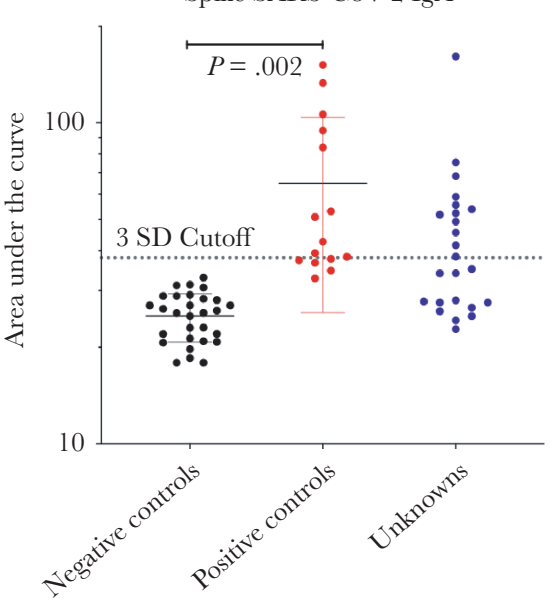

Spike SARS-CoV-2 IgG3

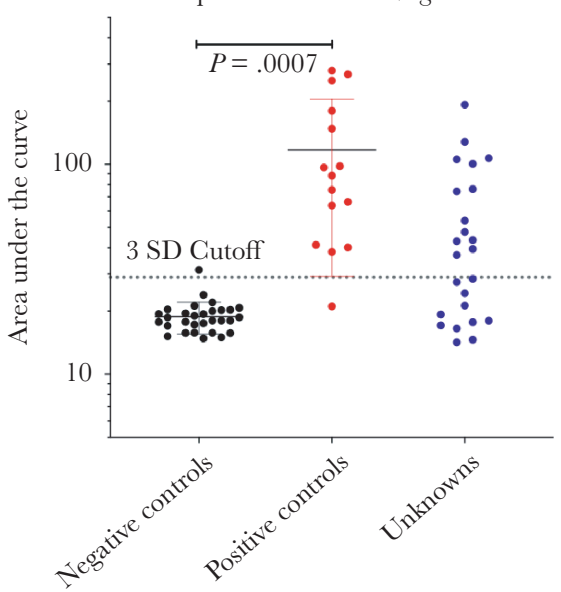

C

NP SARS-GoV-2 IgG

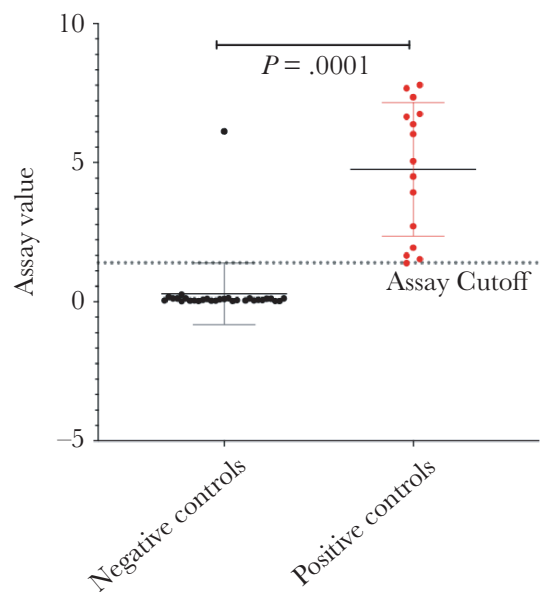

NP SARS-GoV-2 IgG

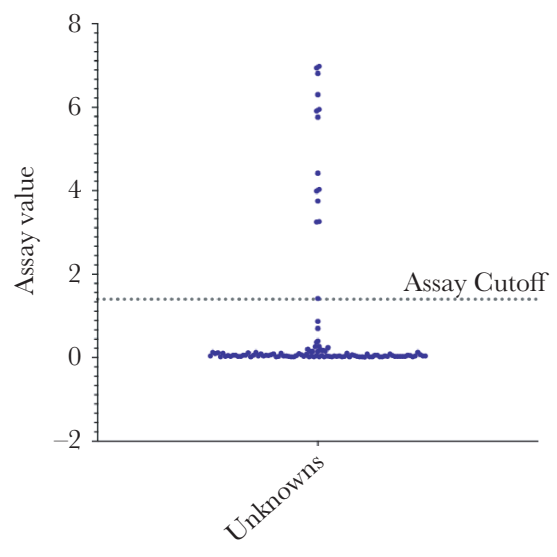

Figure 2. Comparison of ELISA antibody isotype performance. A, IgM antibody recognition of SARS-CoV-2 RBD and spike proteins of all serum samples. B, IgG1, IgG3, and IgA antibody recognition of spike protein of all samples. Mean \pm SD, Student $t$ tests for comparisons of mean of groups. Dotted line represents cutoff of $3 S D$ from the mean of the negative control samples to designate "positive" antibody samples. C, IgG SARS-CoV-2 Abbott NP chemiluminescent microparticle immunoassay results, assay quantitative measurement output, > 1.39 = positive result. Abbreviations: ELISA, enzyme-linked immunosorbent assay; IgG, immunoglobulin G; NP, nucleocapsid protein; RBD, receptor-binding domain; SARS-CoV-2, severe acute respiratory syndrome coronavirus 2. 
A

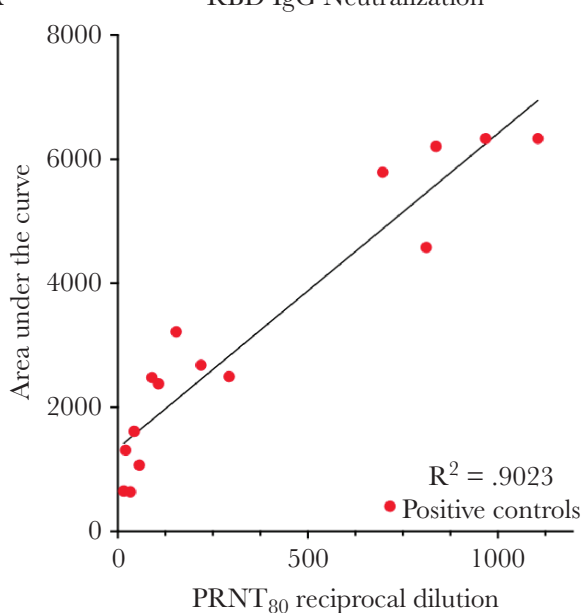

NP IgG Neutralization

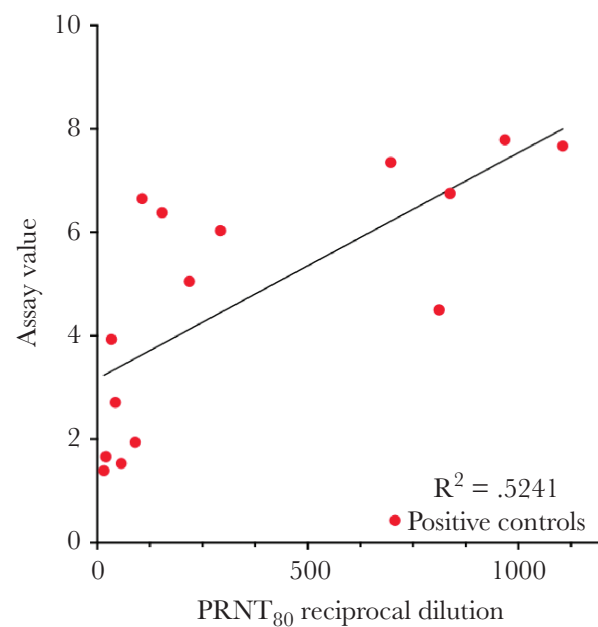

B

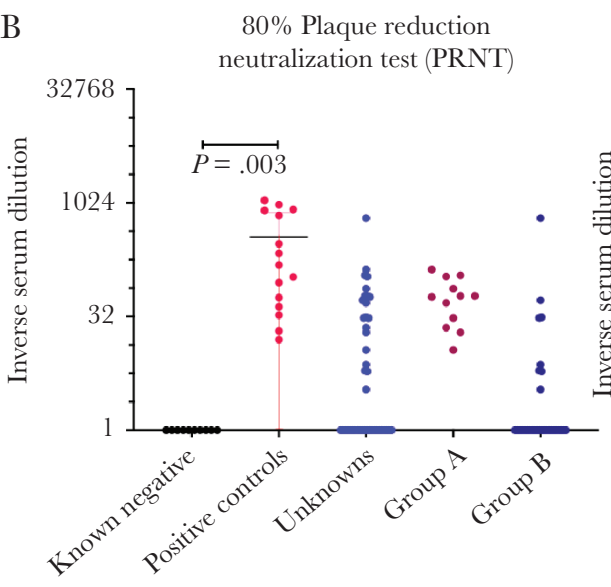

Spike IgG Neutralization

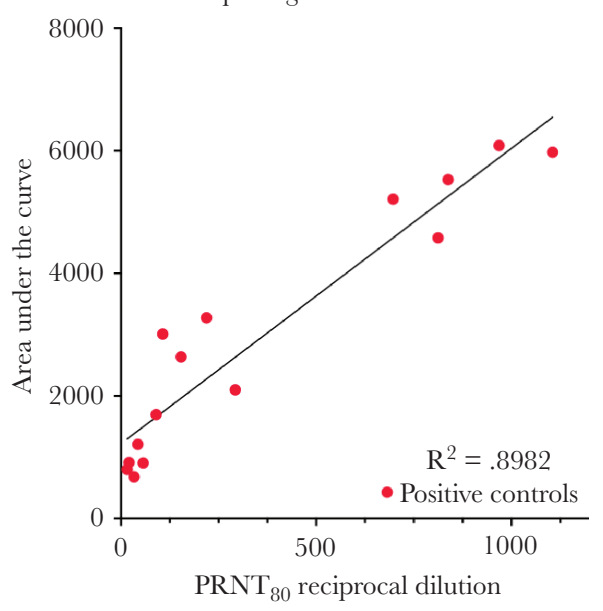

Spike IgG Neutralization

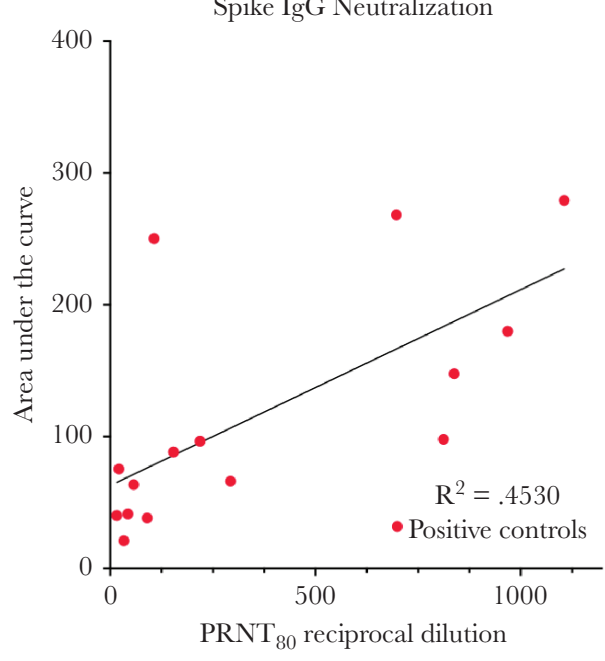

Positive $80 \%$ PRNT samples

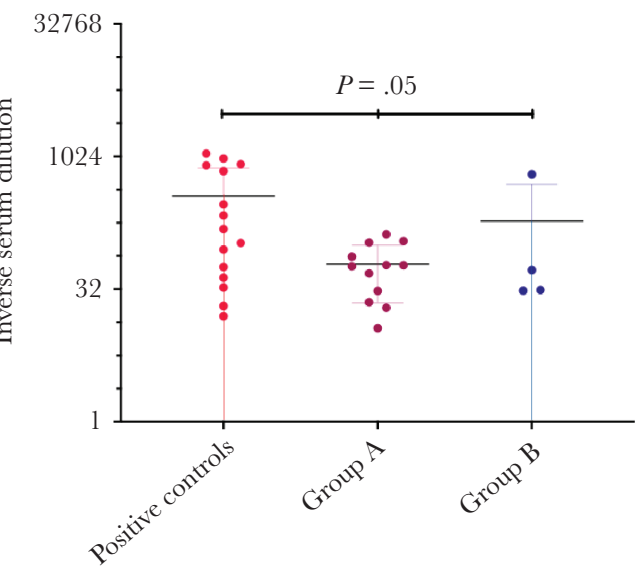

Figure 3. PRNT results. $A$, Linear regression analyses correlating magnitude of neutralization PRNT 80 to magnitude of ELISA serum: spike $\operatorname{lgG}, \mathrm{RBG} \operatorname{lgG}, \mathrm{NP} \operatorname{lgG}$, and spike IgG3. $B$, PRNT $_{80}$ results for all samples and for those meeting neutralization criteria. Mean \pm SD, Student $t$ tests for comparisons of mean of 2 groups and ANOVA analyses for difference among means of 3 groups. Abbreviations: ANOVA, analysis of variance; ELISA, enzyme-linked immunosorbent assay; lgG, immunoglobulin G; NP, nucleocapsid protein; PRNT $_{80}$, 80\% plaque reduction neutralization test; RBD, receptor-binding domain; SARS-CoV-2, severe acute respiratory syndrome coronavirus 2. 
including RBD IgG, spike IgG3, and NP IgG. Figure 4A and 4B summarize and connect the ELISA and PRNT data. The $R^{2}$ correlation between the magnitude of specific ELISA platform antibody detection and neutralization strength is highest for RBD and spike IgG. PRNT percent agreement was calculated for each platform as the number of subjects positive by specific ELISA platforms that were also positive for neutralization for controls samples and unknowns; RBD IgG and spike IgG3 had the highest percent agreement with PRNTs across all cohorts. Subject misidentifications are discordant results between specific ELISA platforms and neutralization not accounted for by the PRNT percent agreement calculations. The spike IgG3 platform had the fewest number of misidentifications followed by NP IgG and RBD IgG. Figure 4B compares the top 3 performing assays across all measures of performance, including RBD IgG, spike IgG3, and NP IgG. Overall, the spike IgG3 assay demonstrated the highest accuracy for identifying serologically positive individuals with detectable neutralizing antibody activity; NP IgG and RBD IgG platforms were slightly inferior in their ability to predict neutralization in our sample set.
Sequential ELISA assay testing platforms have been proposed to increase sensitivity and specificity of SARS-CoV-2 antibody testing [28, 29]. Figure 5 compares sequential ELISA assays using sensitivity, specificity, PRNT agreement, and PRNT misidentifications. No 2 sequential ELISA assays outperformed spike IgG3.

Using detectable neutralization to identify positive unknown samples, we estimated the prevalence of individuals in the Seattle area with neutralizing antibodies against SARS-CoV-2 as of March-April 2020 (Figure 6). Figure 6A is a map of the greater Seattle area with subjects designated by zip code. Figure $6 \mathrm{~B}$ shows the number of positives identified from group A and B as well as those that reported symptoms. Prevalence was estimated using a weighted logistic regression model adjusting for age and sex. The prevalence of individuals with SARS-CoV-2 antibodies at the time of this study was estimated at $3.5 \%$ with a $95 \%$ CI of $1.3 \%-7.3 \%$. Comparison of exposed and unexposed cohorts shows a significant increase in the frequency of detection of neutralizing

\begin{tabular}{|c|c|c|c|c|c|c|}
\hline & PRNT & Spike IgG3 & $\begin{array}{c}\text { RBD IgG } \cdot \cdot> \\
\text { Spike IgG }\end{array}$ & $\begin{array}{l}\text { RBD IgG } \cdots> \\
\text { Spike IgG3 }\end{array}$ & $\begin{array}{c}\text { RBD IgG } \cdots> \\
\text { NP IgG }\end{array}$ & $\begin{aligned} \text { Spike IgG3 } & \cdots> \\
\text { NP IgG } & \end{aligned}$ \\
\hline Sensitivity & $100 \%$ & $93 \%$ & $67 \%$ & $87 \%$ & $87 \%$ & $87 \%$ \\
\hline Specificity & $100 \%$ & $97 \%$ & $97 \%$ & $97 \%$ & $97 \%$ & $93 \%$ \\
\hline PRNT \% Agreement - Unknowns & $100 \%$ & $94 \%$ & $88 \%$ & $88 \%$ & $88 \%$ & $88 \%$ \\
\hline PRNT \% Agreement - Controls & $100 \%$ & $92 \%$ & $75 \%$ & $83 \%$ & $88 \%$ & $83 \%$ \\
\hline PRNT \% Agreement - Total & $100 \%$ & $84 \%$ & $73 \%$ & $77 \%$ & $80 \%$ & $77 \%$ \\
\hline Misidentified PRNTs (MI) & $\mathrm{N} / \mathrm{A}$ & 1 & 3 & 2 & 2 & 2 \\
\hline \multicolumn{7}{|l|}{ Unknowns } \\
\hline \multicolumn{7}{|l|}{1} \\
\hline \multicolumn{7}{|l|}{2} \\
\hline 3 & & & MI & MI & MI & MI \\
\hline \multirow{2}{*}{\multicolumn{7}{|c|}{$\frac{4}{5}$}} \\
\hline & & & & & & \\
\hline \multicolumn{7}{|l|}{6} \\
\hline 7 & & MI & MI & MI & MI & MI \\
\hline 8 & & & MI & & & \\
\hline \multicolumn{7}{|l|}{9} \\
\hline \multicolumn{7}{|l|}{10} \\
\hline \multicolumn{7}{|l|}{11} \\
\hline \multicolumn{7}{|l|}{12} \\
\hline \multicolumn{7}{|l|}{13} \\
\hline \multicolumn{7}{|l|}{14} \\
\hline \multicolumn{7}{|l|}{15} \\
\hline \multicolumn{7}{|l|}{16} \\
\hline \multicolumn{7}{|l|}{17} \\
\hline \multicolumn{7}{|l|}{19} \\
\hline \multicolumn{7}{|l|}{18} \\
\hline 20 & & & & & & \\
\hline
\end{tabular}

Figure 5. Assessment of coupled, sequential antibody tests to increase performance. Testing performance was assessed for 4 possible combinations of ELISA platforms to increase the ability to detect and predict neutralizing antibodies. All the coupled combinations were compared to spike lgG3 as the best performing single ELISA platform. Light color denotes positive ELISA group B subject; dark color denotes positive ELISA, SARS-CoV-2 group A subject; PRNT\% agreement denotes number of ELISA positives in agreement with PRNT positives. MI denotes misidentified outcomes in which incongruent ELISA results with neutralization detection were not encompassed by the \%PRNT agreement calculation. Abbreviations: ELISA, enzyme-linked immunosorbent assay; lgG, immunoglobulin G; NP, nucleocapsid protein; PRNT, plaque reduction neutralization test; RBD, receptor-binding domain; SARS-CoV-2, severe acute respiratory syndrome coronavirus 2. 
A

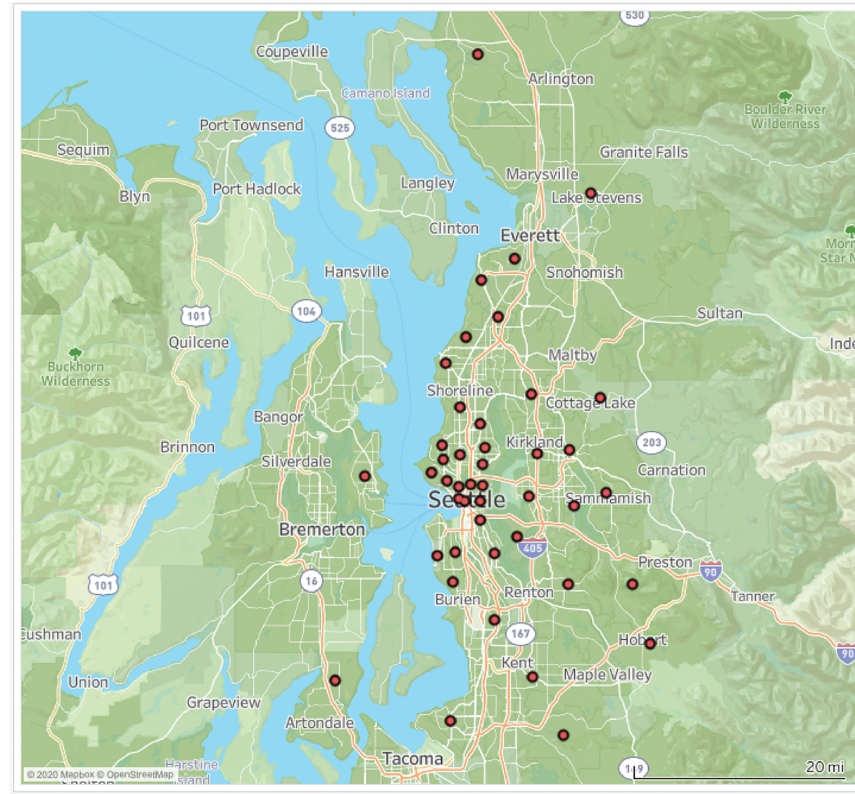

2018 Population

0 to 499

499 to 1640

1640 to 5010

5010 to 18000

18000 to 123000

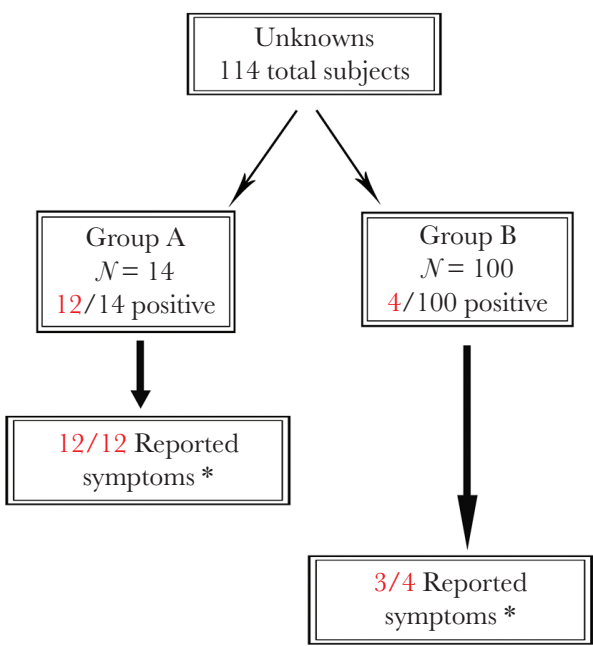

Figure 6. Study subject description and serologic prevalence estimates. A, A map of Seattle with circles denoting the zip codes of the subjects involved in the study. Underlying map colors represent the population density for the areas shown. $B$, Number of subjects, exposure status, and reported symptoms for positive subjects. Group $A$ and $B$ unknown subjects had never been diagnosed with severe acute respiratory syndrome coronavirus 2 (SARS-CoV-2) infection. Group A subjects had close contact with a known infected SARS-CoV-2 individual. Group B subjects had no known exposure to SARS-CoV-2 infected individuals. ${ }^{*}$ Subjects reported symptoms within 5 days of exposure to SARS-CoV-2-positive individuals. ${ }^{* *}$ Subjects reported symptoms over the possible exposure window in the Seattle area (21 January to 15 April 2020).

SARS-CoV-2 antibodies in those with a known exposure to infected individuals.

\section{DISCUSSION}

Our study shows that a 2-tiered testing strategy of ELISA followed by PRNT of positive ELISA samples is the most accurate way to assess humoral immunity to SARS-CoV-2, and that anti-spike IgG3 is the best predictor of presence of neutralizing antibodies. We completed comprehensive analyses of multiple ELISA SARS-CoV-2 platforms coupled with the gold standard of viral neutralization testing to determine a testing strategy most likely to identify true positives and most predictive of SARS-CoV-2 neutralization. Relying on results from a single analysis of testing performance or testing only in control populations did not provide enough information to assess test performance. We found that having paired neutralization studies in control and unknown populations was key to interpreting results of the ELISAs for SARS-CoV-2.

Three ELISA platforms are top performers when we prioritize high sensitivity and specificity, high PRNT agreement, and low misidentification of subjects: RBD IgG, spike IgG3, and NP IgG. Spike IgG3 surpasses RBD IgG because of its high sensitivity and specificity as well as its superior prediction of neutralization in our unknown population (misidentified $=1 / 20$ vs $2 / 20$ ). Of note, the $R^{2}$ for RBD IgG was higher compared to the lower correlation for spike IgG3. However, this difference is challenging to interpret given the finding in other studies with variable and waning correlation with RBD IgG to neutralization ( $R^{2}$ ranging from 0.5 to 0.8 ) when assessed in larger positive control groups $[14,23,24]$. Our positive controls were collected around the same time from infection onset and may explain the high correlation. In assessing unknown populations with our platforms, we found that despite the higher correlation with PRNT, RBD IgG performed less well at predicting neutralization compared to spike IgG3. With the variable reported values of $R^{2}$ for RBD and spike platforms, this outcome measure does not appear to be a dependable measurement of performance for SARS-CoV-2 ELISA platforms to predict neutralization.

Across the ELISA platforms investigated, our data support the use of spike IgG3 as the best initial screening test to predict neutralization but with the important caveat of our limited sample size. Many of the parameters of test performance were similar for RBD IgG, spike IgG3, and NP IgG. However, spike IgG3 edged out both NP and RBD IgG with the ability to predict neutralization (\% PRNT agreement and misidentification). Expanded studies comparing these 3 platforms with larger sample sizes would be beneficial. RBD and NP IgG platforms already have FDA emergency approval, with the NP IgG platform having the highest sensitivity and specificity as well as the most comprehensive validation $[34,35]$. The NP IgG assay 
is likely to be limited in its ability to predict antibody neutralization activity due to internal NP localization within the intact virion being inaccessible to antibodies in vivo. This consideration is especially relevant for vaccine responses, as current vaccines being launched for human application are designed to generate immunity against the virion spike protein $[35,36]$. Spike IgG3 platforms have not been developed but represent a highly promising platform given that IgG3 isotypes are thought to be more effective at viral neutralization compared to other IgG subtypes $[25,26]$.

Classic PRNTs are expensive and time consuming due to laboratory biosafety requirements. However, development of pseudovirus systems have demonstrated high correlation with the SARS-CoV-2 PRNT assay, which would allow for highthroughput sample analysis of neutralizing antibodies [14, 37, 38]. Coupling of a pseudovirus system with spike IgG3 ELISA would provide an accurate and practical 2-tiered testing method to use within a standard clinical laboratory to assess for possible correlates of immunity against SARS-CoV-2.

Lastly, we estimated the prevalence of individuals with detectable SARS-CoV-2 neutralizing antibodies in the Seattle area. Our sample size is very small and therefore unavoidably biased. The United States Center for Disease Control (CDC) reported a prevalence of $1.13 \%$ in samples obtained from the same time frame but their study suffers from sampling bias given the unknown population utilized [39]. The CDC study prevalence estimate could be either an over- or underestimate. We propose that the $95 \%$ CI $(1.3 \%-7.3 \%)$ range found in this study offers a more accurate estimate of SARS-CoV-2 prevalence in our community compared to a point prevalence estimate.

In this study, we show how neutralizing assays serve as a check on the accuracy of SARS-CoV-2 antibody screening tests. Rapid, significant contributions by scientists worldwide have produced serologic SARS-CoV-2 data that have been consistent in one important way: variability. Our study confirms this high variability in serologic assay performance and provides further data that no single serologic assay provides perfect prediction for viral neutralizing ability. Two-tiered testing would allow parsing of subjects into groups important for further study in the setting of large-scale vaccine trials: (1) those with positive ELISA antibody detection and confirmed neutralization, and (2) those with positive ELISA antibody detection but no evidence of neutralization. Group 1 would allow tracking of subjects with known neutralization titers for evidence of reinfection versus possible protective immunity. Group 2 would allow study for increased identification of true false positives versus individuals that do not develop functional and/or lasting neutralizing antibodies. Therefore, a 2-tiered testing strategy in which a high-throughput pseudovirus assay is coupled to an accurate serologic assay would provide key data to identify subjects with possible protective immunity to SARS-CoV-2, and to assess vaccine efficacy. At the time of this study we were unable to evaluate sera from vaccine clinical trial studies. However, mRNA vaccines against the spike protein have demonstrated detectable antibodies with RBD and spike IgG platforms similar to the platforms investigated here [40-42].

To our knowledge this is the first study to comprehensively assess serologic assay performance in an unknown cohort across antigen and antibody isotype comparisons to determine a practical and accurate method to determine the presence of SARS-CoV-2 neutralizing antibodies. By coupling ELISA with virus neutralization assessment, we were able to determine the accuracy of testing based on the key functional outcome of viral neutralization. Pseudoneutralization or FRNT assays provide a comparable test of neutralization to PRNTs and could be rapidly developed with a new spike IgG3 or an existing ELISA platform for 2-tiered testing [14, 37, 43]. Our study provides compelling evidence that a 2-tiered testing scheme, ideally consisting of assessment of spike IgG3 antibodies and virus neutralization analysis, optimally facilitates staging patients for SARS-CoV-2 antibody prevalence and a possible correlate of humoral immune protection to monitor clinical status and vaccine efficacy.

\section{Supplementary Data}

Supplementary materials are available at The Journal of Infectious Diseases online. Consisting of data provided by the authors to benefit the reader, the posted materials are not copyedited and are the sole responsibility of the authors, so questions or comments should be addressed to the corresponding author.

\section{Notes}

Acknowledgments. We thank Keith Jerome and Gregory Pepper for SARS-CoV-2 NP ELISA; Neil King and his laboratory members for providing soluble trimeric SARS-CoV-3 spike protein used in our ELISA assays; and Lauren Rodda for her input on experimental design and analyses.

Financial support. This work was supported by the National Institutes of Health (grant numbers AI148684, AI151698, and AI145296); and the University of Washington funds to the Center for Innate Immunity and Immune Disease.

Potential conflicts of interest. All authors: No reported conflicts of interest. All authors have submitted the ICMJE Form for Disclosure of Potential Conflicts of Interest. Conflicts that the editors consider relevant to the content of the manuscript have been disclosed. The authors declare no conflict of interest.

\section{References}

1. Kohmer N, Westhaus S, Rühl C, Ciesek S, Rabenau HF. Brief clinical evaluation of six high-throughput SARS-CoV-2 IgG antibody assays. J Clin Virol 2020; 129:104480.

2. Kohmer N, Westhaus S, Rühl C, Ciesek S, Rabenau HF. Clinical performance of different SARS-CoV-2 IgG antibody tests. J Med Virol 2020; 92:2243-7. 
3. Vandergaast R, Carey T, Reiter S, et al. Development and validation of IMMUNO-COV ${ }^{\mathrm{sN}}$ : a high-throughput clinical assay for detecting antibodies that neutralize SARS-CoV-2. bioRxiv, doi: 10.1101/2020.05.26.117549, 27 May 2020, preprint: not peer reviewed.

4. Breuer J, Schmid DS, Gershon AA. Use and limitations of varicella-zoster virus-specific serological testing to evaluate breakthrough disease in vaccinees and to screen for susceptibility to varicella. J Infect Dis 2008; 197(Suppl 2):S147-51.

5. Gershon AA, Larussa P, Steinberg S. Detection of antibodies to varicella-zoster virus using a latex agglutination assay. Clin Diagn Virol 1994; 2:271-7.

6. Ludwig B, Kraus FB, Allwinn R, Keim S, Doerr HW, Buxbaum S. Loss of varicella zoster virus antibodies despite detectable cell mediated immunity after vaccination. Infection 2006; 34:222-6.

7. Hammond O, Wang Y, Green T, et al. The optimization and validation of the glycoprotein ELISA assay for quantitative varicella-zoster virus (VZV) antibody detection. J Med Virol 2006; 78:1679-87.

8. Cortese MM, Barskey AE, Tegtmeier GE, et al. Mumps antibody levels among students before a mumps outbreak: in search of a correlate of immunity. J Infect Dis 2011; 204:1413-22.

9. O’Shea S, Best JM, Banatvala JE. Viremia, virus excretion, and antibody responses after challenge in volunteers with low levels of antibody to rubella virus. J Infect Dis 1983; 148:639-47.

10. Wilson SE, Deeks SL, Hatchette TF, Crowcroft NS. The role of seroepidemiology in the comprehensive surveillance of vaccine-preventable diseases. CMAJ 2012; 184:E70-6.

11. Chen RT, Markowitz LE, Albrecht P, et al. Measles antibody: reevaluation of protective titers. J Infect Dis 1990; 162:1036-42.

12. Samb B, Aaby P, Whittle HC, et al. Serologic status and measles attack rates among vaccinated and unvaccinated children in rural Senegal. Pediatr Infect Dis J 1995; 14:203-9.

13. Hatchette TF, Scholz H, Bolotin S, et al. Calibration and evaluation of quantitative antibody titers for measles virus by using the BioPlex 2200. Clin Vaccine Immunol 2017; 24:e00269-16.

14. Suthar MS, Zimmerman MG, Kauffman RC, et al. Rapid generation of neutralizing antibody responses in COVID19 patients. Cell Rep Med 2020; 1:100040.

15. Long QX, Liu BZ, Deng HJ, et al. Antibody responses to SARS-CoV-2 in patients with COVID-19. Nat Med 2020; 26:845-8.

16. To KK, Hung IF, Ip JD, et al. COVID-19 re-infection by a phylogenetically distinct SARS-coronavirus-2 strain confirmed by whole genome sequencing [published online ahead of print 25 August 2020]. Clin Infect Dis doi: 10.1093/ $\mathrm{cid} / \mathrm{ciaa} 1275$.
17. Tillett R, Sevinsky J, Hartley P, et al. Genomic evidence for reinfection with SARS-CoV-2: a case study. Lancet Infect Dis 2020; 21:52-8.

18. Murillo-Zamora E, Mendoza-Cano O, Delgado-Enciso I, Hernandez-Suarez CM. Predictors of severe symptomatic laboratory-confirmed SARS-COV-2 reinfection. medRxiv, doi:10.1101/2020.10.14.20212720, 18 October 2020, preprint: not peer reviewed.

19. Beavis KG, Matushek SM, Abeleda APF, et al. Evaluation of the EUROIMMUN Anti-SARS-CoV-2 ELISA assay for detection of IgA and IgG antibodies. J Clin Virol 2020; 129:104468.

20. Montesinos I, Gruson D, Kabamba B, et al. Evaluation of two automated and three rapid lateral flow immunoassays for the detection of anti-SARS-CoV-2 antibodies. J Clin Virol 2020; 128:104413.

21. Van Elslande J, Houben E, Depypere M, et al. Diagnostic performance of 7 rapid $\mathrm{IgG} / \mathrm{IgM}$ antibody tests and the Euroimmun $\operatorname{IgA} / \mathrm{IgG}$ ELISA in COVID-19 patients. Clin Microbiol Infect 2020; 26:1082-7.

22. Jääskeläinen AJ, Kuivanen S, Kekäläinen E, et al. Performance of six SARS-CoV-2 immunoassays in comparison with microneutralisation. J Clin Virol 2020; 129:104512.

23. Muecksch F, Wise H, Batchelor B, et al. Longitudinal analysis of clinical serology assay performance and neutralising antibody levels in COVID19 convalescents. medRxiv, doi: 10.1101/2020.08.05.20169128, 6 August 2020, preprint: not peer reviewed.

24. Wu F, Wang A, Liu M, et al. Neutralizing antibody responses to SARS-CoV-2 in a COVID-19 recovered patient cohort and their implications. medRxiv, doi:10.110 1/2020.03.30.20047365, 20 April 2020, preprint: not peer reviewed.

25. Irani V, Guy AJ, Andrew D, Beeson JG, Ramsland PA, Richards JS. Molecular properties of human IgG subclasses and their implications for designing therapeutic monoclonal antibodies against infectious diseases. Mol Immunol 2015; 67:171-82.

26. Papadea C, Check IJ. Human immunoglobulin G and immunoglobulin G subclasses: biochemical, genetic, and clinical aspects. Crit Rev Clin Lab Sci 1989; 27:27-58.

27. Xiang F, Wang X, He X, et al. Antibody detection and dynamic characteristics in patients with coronavirus disease 2019. Clin Infect Dis 2020; 71:1930-4.

28. Stadlbauer D, Amanat F, Chromikova V, et al. SARS-CoV-2 seroconversion in humans: a detailed protocol for a serological assay, antigen production, and test setup. Curr Protoc Microbiol 2020; 57:e100.

29. Dingens AS, Crawford KHD, Adler A, et al. Serological identification of SARS-CoV-2 infections among children visiting a hospital during the initial Seattle outbreak. Nat Commun 2020; 11:4378. 
30. Walls AC, Park YJ, Tortorici MA, Wall A, McGuire AT, Veesler D. Structure, function, and antigenicity of the SARS-CoV-2 spike glycoprotein. Cell 2020; 181: 281-92.e6.

31. Chao CC, Zhang Z, Belinskaya T, et al. An ELISA assay using a combination of recombinant proteins from multiple strains of Orientia tsutsugamushi offers an accurate diagnosis for scrub typhus. BMC Infect Dis 2017; 17:413.

32. Hartman H, Wang Y, Schroeder HW Jr, Cui X. Absorbance summation: a novel approach for analyzing highthroughput ELISA data in the absence of a standard. PLoS One 2018; 13:e0198528.

33. Erasmus JH, Khandhar AP, Walls AC, et al. Single-dose replicating RNA vaccine induces neutralizing antibodies against SARS-CoV-2 in nonhuman primates. bioRxiv, doi: 10.1101/2020.05.28.121640, 28 May 2020, preprint: not peer reviewed.

34. Bryan A, Pepper G, Wener MH, et al. Performance characteristics of the Abbott Architect SARS-CoV-2 IgG assay and seroprevalence in Boise, Idaho. J Clin Microbiol 2020; 58:e00941-20.

35. MeschiS, Colavita F, Bordi L, et al; INMICovid-19Laboratory Team. Performance evaluation of Abbott Architect SARSCoV-2 IgG immunoassay in comparison with indirect immunofluorescence and virus microneutralization test. J Clin Virol 2020; 129:104539.
36. Weiss SR, Leibowitz JL. Coronavirus pathogenesis. Adv Virus Res 2011; 81:85-164.

37. Muruato AE, Fontes-Garfias CR, Ren P, et al. A highthroughput neutralizing antibody assay for COVID-19 diagnosis and vaccine evaluation. Nat Commun 2020; 11:4059.

38. Tan CW, Chia WN, Qin X, et al. A SARS-CoV-2 surrogate virus neutralization test based on antibody-mediated blockage of ACE2-spike protein-protein interaction. Nat Biotechnol 2020; 38:1073-8.

39. Havers FP, Reed C, Lim T, et al. Seroprevalence of Antibodies to SARS-CoV-2 in 10 Sites in the United States, March 23-May 12, 2020. JAMA Intern Med 2020.

40. Anderson EJ, Rouphael NG, Widge AT, et al; mRNA-1273 Study Group. Safety and immunogenicity of SARS-CoV-2 mRNA-1273 vaccine in older adults. N Engl J Med 2020; 383:2427-38.

41. Jackson LA, Anderson EJ, Rouphael NG, et al; mRNA-1273 Study Group. An mRNA vaccine against SARS-CoV-2preliminary report. N Engl J Med 2020; 383:1920-31.

42. Widge AT, Rouphael NG, Jackson LA, et al. Durability of responses after SARS-CoV-2 mRNA-1273 vaccination [published online ahead of print 3 December 2020]. N Engl J Med doi: 10.1056/NEJMc2032195.

43. Crawford KHD, Eguia R, Dingens AS, et al. Protocol and reagents for pseudotyping lentiviral particles with SARS-CoV-2 spike protein for neutralization assays. Viruses 2020; 12:513. 